\title{
A wide field survey of Sagittarius dSph. Data and tools for the study of the Sgr Tidal Stream
}

\author{
M. Correnti ${ }^{1} \dagger$, M. Bellazzini ${ }^{2}$, \\ F. R. Ferraro ${ }^{1}$ and L. Monaco ${ }^{3}$ \\ ${ }^{1}$ Dipartimento di Astronomia, Universitá di Bologna, Italia \\ email: matteo.correnti@studio.unibo.it \\ ${ }^{2}$ INAF - Osservatorio Astronomico di Bologna, Italia \\ ${ }^{3}$ European Southern Observatory, Santiago, Chile
}

\begin{abstract}
We present deep B,V,I photometry of three wide fields within the core of the Sagittarius dwarf spheroidal galaxy (Sgr dSph). We provide accurate templates for some well identified sequences of the CMD of the galaxy (Blue HB, Red HB, etc.) as tools to recognize portion of the tidal Stream detected by various surveys, to compare the stellar populations in the main body of Sgr and in its Stream, and to derive accurate distances to Stream stars.
\end{abstract}

\section{Introduction}

The Bologna wide field survey of the Sagittarius dwarf spheroidal (Sgr dSph) collects deep BVI photometry of four wide fields within the core of the Sgr galaxy; it covers a total area of $2.5 \mathrm{deg}^{2}$ (with an increase of a factor $\sim 23$ respect the previous largest survey) and reaches $V \sim 23.5$. Here we briefly present the first scientific results of the survey and we outline some possible future applications.

\section{Main Results}

In Bellazzini et al. (2006) we have derived robust constraints on the mean age of the stellar populations that dominates the galaxy (POP A). Using three different sets of theoretical isochrones in the metallicity range $-0.4 \leqslant[M / H] \leqslant-0.7$ and taking into consideration distance moduli in the range $16.90 \leqslant(m-M)_{0} \leqslant 17.20$ we find that the mean age of POP A is larger than $5 \mathrm{Gyr}$, and the best fit value is age $=8.0 \pm 1.5 \mathrm{Gyr}$. Since POP A provides the vast majority of M giants that traces the stream of Sgr dSph all over the sky, our estimate resolve the so called "M giant conundrum" (Majewski et al. 2003). The time needed by the $M$ giants that currently populate the Stream to diffuse within the main body of Sgr and to reach the extremes of the tidal tails once torn apart from the parent galaxy $(\approx 3-4 \mathrm{Gyr})$ can be easily accomodated into the time lapsed since their birth $(\approx 5.5-9.5 \mathrm{Gyr})$.

In Bellazzini et al. (2006), thanks to a quantitative comparison between the Horizontal Branch morphology in the core of Sgr and in a wide field sampling a portion of its tidal stream (taken from the SDSS, Newberg et al. 2002), we find that the Blue Horizontal Branch (BHB) stars in that part of the Stream are five times more abundant than in the Sgr core, relative to Red Clump stars. The difference in the ratio of BHB to RC stars between the two fields is significant at $\geqslant 4.8 \sigma$ level. This indicates that the old

$\dagger$ Present address: INAF-OABo, via Ranzani 1, 40137, Bologna, Italia. 


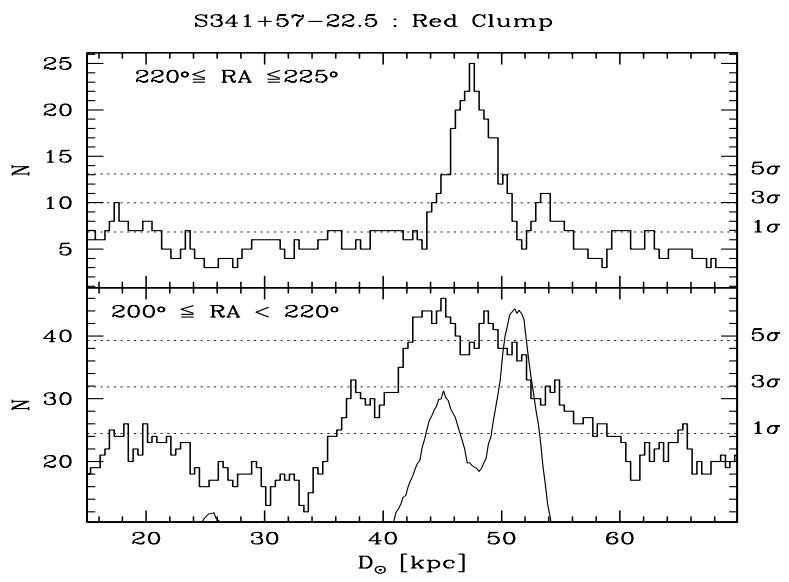

Figure 1. Distance distribution of Color-selected candidate RC stars in a portion of the Sgr Stream. Distances have been assigned by photometric parallax, assuming $(m-M)_{0}=17.10$ for Sgr and $V_{R C}^{S g r}=17.83$, as measured from our CMD. Obvious and very significant peaks in the distance distribution along the considered l.o.s. are easily detected. In the lower panel we show a case in which we detect a bifurcation in the distance distribution: this correspond to the crossing of two concentric wraps of the Stream, as predicted by current N-body models of Sgr in this direction (thin curve, spherical model by Law et al. 2005)

and metal-poor population of Sgr was preferentially stripped from the galaxy in pasti peri-Galactic passages with respect to the intermediate-age metal-rich population that presently dominates the bound core of Sgr.

\section{Future Works}

An obvious application of the comparison between our CMD of the core of Sgr and the CMDs of portions of the Stream (f.i. taken from the SDSS) is to obtain distance estimates to the Stream itself by photometric parallax. Several CMD features can be used for this purpose: the $\mathrm{RC}$, the $\mathrm{BHB}$, the Main Sequence. In many cases all of the these distance indicators can be used simultaneously. Moreover, multiband photometry may provide more than one indipendent distance estimate from the same indicators (as, for example, the mean $\mathrm{V}$ and I magnitude of the $\mathrm{RC}$ ). We have verified that existing photometric trasformations between the SDSS system and Johnson-Cousins BVI are sufficently accurate to permit fruitful comparisons. The analysis of the distance distribution of the Northern part of the Sgr Stream is currently in progress and an example of a possible approach to the problem is shown in Fig. 1.

This research is supported by INAF through the grant PRIN05 1.06.08.03.

\section{References}

Bellazzini, M., Correnti, M., Ferraro, F.R., Monaco, 1., \& Montegriffo, P., 2006, A\&A 446, L1-L4

Bellazzini, M., Correnti, M., Ferraro, F.R., \& Monaco, L., 2006, A\&A 457, L21-L24

Law, D.R., Johnston, K.V., \& Majewski, S.R., 2005, ApJ, 619, 807

Majewski, S.R., Skrutskie, M.F., Weinberg, M.D., \& Ostheimer, J.C., 2003, ApJ, 599, 1082

Newberg, H.J., et al. 2002, ApJ, 569, 245 